\title{
Editorial
}

\section{Misuse of "coronary heart disease"}

Precision and non-ambiguity are paramount adjuncts of word usage and scientific communication, particularly when the term coronary heart disease (CHD) is in common use epidemiologically and as an end point in clinical trials. CHD, as non-sensical as "femoral leg disease", may have led non-medical epidemiologists to consider it a specific disease when this is pathologically false.

"Disease" can be used in a general sense to indicate a diseased state, the antithesis of health. "A disease" should signify a specific disease entity (such as atherosclerosis) and should not be used loosely in reference to symptoms (angina pectoris, headache), signs (fever, hypertension), laboratory findings (hypercholesterolaemia), non-specific complications (embolism, haemorrhage, ischaemia, necrosis), pathological lesions (gangrene, aneurysm, infarct, haematoma), abnormal functional states (obstructive lung disease) or collective terms for pathological states or diseases related to an organ (renal disease, cerebrovascular disease, CHD). Each exclusion is a manifestation of many specific diseases varying aetiologically and pathogenetically. Some are non-specific clinical diagnoses, not specific disease entities. Loose usage cannot be condoned.

Disease classification based on aetiological or pathogenetic mechanisms is preferable to classification based on anatomical sites, functional changes or non-specific pathological lesions. Aneurysm and subarachnoid haemorrhage cannot be treated clinically or epidemiologically as single disease entities for each is a non-specific complication of many diseases (even trauma). Pooling diverse diseases that cause aneurysms for epidemiological or therapeutic study is unwise and this holds true for myocardial ischaemia that cannot be used to infer the presence of coronary atherosclerosis of any given severity nor does its experimental production indicate that the underlying arterial lesion is atherosclerotic. CHD is an imprecise, nonspecific, clinical collective diagnostic term for a heterogeneous group of pathological states and diseases producing myocardial ischaemia. ${ }^{1}$ It is not a specific disease although used as such in epidemiology.

Initially, Framingham investigators considered CHD was not a definable epidemiological entity and excluded CHD vital statistics from epidemiological use even for coronary atherosclerosis, ${ }^{2}$ although it was held legitimate to define CHD in terms of clinical syndromes (angina pectoris, myocardial infarction, sudden death). These syndromes were said to vary in reliability as indicators of CHD. It was emphasised that the epidemiology of CHD was not the epidemiology of coronary atherosclerosis, ${ }^{3}$ the investigators being aware that pathological differences in subjects with and without clinical CHD were of degree rather than kind, although atherosclerosis severity was never assessed ${ }^{3}$ nor was the degree of overlap known. Subsequently, these clinical syndromes were amalgamated as $\mathrm{CHD}$, although none was any more specific for atheroscle- rosis of indeterminate severity than the imprecise composite CHD.

In the International Statistical Classification of Diseases, ischaemic heart disease (IHD) encompasses clinical and pathological manifestations of myocardial ischaemia and its complications (angina pectoris, myocardial infarction, cardiac aneurysm, and ischaemic myocardiopathy) without reference to either the severity of ischaemia or the underlying coronary artery diseases responsible for the impaired blood supply. Specifying ischaemia, IHD is more explicit, is less likely to be confused with coronary atherosclerosis per se, and is therefore preferable to the more commonly used CHD. As the implied meanings are identical, the more appropriate IHD should be used.

Some clinicians and authors may have different definitions for IHD but it would avoid confusion and misrepresentation if IHD encompassed myocardial ischaemia over the full range of severity from mild silent ischaemia to infarction. Understandably, the underlying coronary arterial pathology cannot always be defined ante mortem, and for the foreseeable future in such cases "CHD" or "IHD" will continue in use unless it is clear some nonatherosclerotic disease is primarily responsible.

Approximately $10 \%$ of IHD cases are attributable to non-atherosclerotic diseases, which are many and varied. ${ }^{14}$ When atherosclerotic in origin, several associated pathological conditions can adversely affect atherosclerosis during its insidious progression over decades. Extraneous factors can initiate or contribute to its eventual clinical or fatal manifestations and sometimes concomitant diseases are superimposed. Under such circumstances IHD cannot be equated with coronary atherosclerosis. Every medical graduate should be cognisant of their differences in pathology and pathogenesis. Inappropriately defined as synonymous, ${ }^{5}$ they are currently used interchangeably and IHD epidemiology is equated with that of atherosclerosis. ${ }^{4}$

The basic epidemiological paradigm of comparing diseased subjects with healthy subjects is misused because atherosclerosis is ubiquitous. As Framingham investigators stated, ${ }^{3}$ the difference between individuals is a difference in atherosclerosis severity that cannot be measured during life. Often resorted to as a way out of the dilemma, angiography displays the silhouette of the lumen and is an insensitive method for detecting early atherosclerosis or diffuse involvement of entire vessels or for assessing severity. Measurements of intima-media thickness constitute a balance between stenosing intimal thickening and the extent of media thinning with yield of media and adventitia. These procedures do not assess qualitative mural changes characteristic of the mural fragility responsible for intimal tearing, ulceration, aneurysmal dilatation, etc. Pathologists are unlikely to agree on a suitable necropsy parameter to grade severity when choice encompasses lipid content, histological assessment, degree of stenosis, vascular fragility or surface area involved. Epidemiological parameters should be defined precisely and accurately measured. This does 
not happen in IHD epidemiology. The situation is aggravated by variation in severity from site to site, and local variations in arterial topography and flow affect the site specific rates of progression. ${ }^{6}$ The clinical incidence of non-pathognomonic IHD is not a precise end point nor a sensitive surrogate for undefined severity of universal coronary atherosclerosis. This fundamental, incontrovertible flaw is of sufficient magnitude to invalidate much IHD epidemiology. Lamentably the misrepresentation is aggravated by misdiagnosis of IHD which, when monitored by necropsy, amounts to $\pm 30 \%$ varying within wide limits from study to study. ${ }^{7} \mathrm{Up}$ to $70 \%$ of cardiac ischaemic attacks during daily activities are silent ${ }^{8}$ and $10-20 \%$ of subjects with angina pectoris have normal or nearly normal coronary angiograms. ${ }^{9}$ Monocausal, national IHD mortality rates in wide use epidemiologically are crude at best and provide fallacious data for scientific purposes. ${ }^{10}$ Furthermore, extrapolations from these population statistics to individuals are invalid.

The consequences of this misuse of IHD or CHD are serious. Risk factors are statistical associations with IHD and are irrelevant to atherogenesis when IHD is nonatherosclerotic. Risk factors of atherosclerotic IHD relate to its end stage rather than its genesis and are unhelpful in determining cause. It amounts to seeking the cause by studying statistical correlations with a non-specific clinical surrogate of atherosclerosis of indefinite severity in pooled subjects, some with non-atherosclerotic IHD and others with IHD as an end stage complication of severe atherosclerosis. Extrapolations from these risk factors to aetiology of atherosclerosis would provide invalid results. Kannel ${ }^{11}$ admitted that none of the major risk factors comply with the soft epidemiological criteria for causation. Current programmes to prevent IHD implicitly assume the validity of the lipid hypothesis, the exclusive underlying concern being lipid metabolism in atherogenesis rather than the role of lipid in the production of myocardial ischaemia of diverse causes, and assessing the effect of an intervention by IHD incidence rather than pathological severity of atherosclerosis.

In summary, IHD is a preferable term to CHD. Both are clinical syndromes pertaining to myocardial ischaemia rather than the severity of ubiquitous coronary atherosclerosis, the rightful parameter for assessment in epidemiology. Consequently, the misuse of IHD incidence as a surrogate for the severity of coronary atherosclerosis needs to be acknowledged and redressed. The wide implications of this error are yet to be fully realised.

Financial support from the Wellington Medical Research Foundation is gratefully acknowledged.

Department of Pathology,

Wellington School of Medicine,

PO Box 7343, Wellington South, New Zealand

1 Waller BF. Atherosclerotic and nonatherosclerotic coronary artery factors in acute myocardial infarction. In: Pepine CJ, ed. Acute myocardial infarction Philadelphia: Davis, 1989:29-104.

Dawber TR, Kannel WB. Coronary heart disease as an epidemiology entity. Am f Pub Health 1963;53:433-7.

3 Dawber TR, Kannel WB, Lyell LP. An approach to longitudinal studies in a community: the Framingham study. Ann NY Acad Sci 1963;107:539-56.

4 Robbins SL, Angell M, Kumar V. Basic pathology, 3rd ed. Philadelphia: Saunders. 1981:289-99.

5 National Heart, Lung and Blood Institute. Arteriosclerosis. Report of the National Heart and Lung Institute Task Force on Arteriosclerosis. Washington: DHEW Publication No (NIH) 78-1526, 1977.

6 Stehbens WE. Atherosclerosis and degenerative diseases of blood vessels. In: Stehbens WE, Lie JT, eds. Vascular pathology. London: Chapman \& Hall, Stehbens WE

7 Stehbens WE. An appraisal of the epidemic rise of coronary heart disease and its decline. Lancet $1987 ; \mathrm{i}: 606-11$.

8 Maseri A, Chierchia S, Davies GJ, et al. Mechanisms of ischemic cardiac pain and silent myocardial ischemia. Am f Med 1985;79(suppl 3A):7-11. pain and silent myocardial ischemia. Am $\mathcal{f}$ Med 1985;79(suppl 3A):7-11.
9 Gazes PC. Angina pectoris: classification and diagnosis. Part 2. Mod Gazes PC. Angina pectoris: classifica
Concepts Cardiovasc Dis 1988;57:25-7.

10 Feinstein AR. Clinical epidemiology. The architecture of clinical research. Philadelphia: Saunders, 1985.

11 Kannel WB. Recent highlights from the Framingham Study. Aust NZ F Med 1976;6:373-86. 